\title{
A retrospective study of the association between Fuchs' endothelial dystrophy and glaucoma
}

\author{
This article was published in the following Dove Press journal: \\ Clinical Ophthalmology \\ 21 October 2014 \\ Number of times this article has been viewed
}

\author{
Greg D Rice' \\ Kristin Wright ${ }^{\prime}$ \\ Steven M Silverstein ${ }^{2}$ \\ 'Kansas City University of Medicine \\ and Biosciences, Kansas City, MO, \\ USA; ${ }^{2}$ Silverstein Eye Centers, Kansas \\ City, MO, USA
}

Objective: The goal of this study was to examine a possible clinical association between Fuchs' endothelial dystrophy (FED) and glaucoma suspect (GS)/ocular hypertension (OHT) or open angle glaucoma (OAG).

Methods: A retrospective chart review was carried out using data from electronic medical records and paper records from a private ophthalmology clinic in Kansas City, MO, USA. The review included 257 patients with FED and 584 randomly selected controls with no history of endothelial dystrophy. Binomial and multinomial regression using generalized estimating equations was used to create models to examine the correlation between FED diagnosis/severity and glaucoma diagnosis/type of glaucoma adjusted for age, sex, presence of diabetes, number of guttae, and intraocular pressure (IOP).

Results: No statistically significant increase in prevalence of either OHT or GS/OHT compared to controls was observed $(P>0.3)$. There was a statistically significant positive correlation between increasing age and IOP with increased glaucoma prevalence $(P<0.05)$. There was also a statistically significant positive correlation between increasing age, IOP and male sex, with increased prevalence of the more severe glaucoma subtype of OAG versus GS/OHT and controls $(P<0.05)$. Increasing severity of FED divided into category 1 and 2 based upon number of guttae was not associated with any significant increase in glaucoma prevalence $(P>0.09)$, and was actually significantly negatively correlated to worsening glaucoma subtype for category 2 FED patients $(P<0.05)$. Diabetes was not associated with the prevalence of either glaucoma or its subtypes of GS/OHT or OAG.

Conclusion: The correlation between FED and glaucoma has been controversial. This study showed no statistically significant association between FED and glaucoma by prevalence or severity of FED as measured by corneal guttae. Further study is needed to determine if a connection between FED and glaucoma does exist, and if so, whether this relationship may impact earlier the detection and treatment of disease.

Keywords: ocular hypertension, open angle glaucoma, cornea guttata, oxidative

\section{Introduction}

Multiple studies have proposed an association between Fuchs' endothelial dystrophy (FED) and the various subsets of glaucoma. This proposed association has been used as a rationale for combining lens extraction with penetrating keratoplasty for Fuchs' endothelial dystrophy patients ${ }^{1}$ and could be the basis for increased glaucoma screening in FED patients. A relationship between these two diseases is plausible and has clinical basis, but remains unconfirmed. The objective of this study was to complete a retrospective chart review of patients with FED and glaucoma to determine if a clinical association exists between the two diseases.

FED is an autosomal dominant corneal disorder of abnormal collagen deposition in Descemet's membrane, a membrane which lies between the endothelial cells and
Correspondence: Kristin Wright Kansas City University of Medicine and Biosciences, 1750 Independence Avenue, Kansas City, MO, 64I06-1453, USA

Email kwright@kcumb.edu 
stroma of the cornea, leading to progressive degeneration of endothelial cells. ${ }^{2}$ Loss of endothelial cells prevents removal of water from the cornea by the Na K-ATPase pumps, causing corneal edema. Corneal edema leads to glare and blurred vision, with ultimate loss of vision. ${ }^{2}$ FED often presents from the fourth through seventh decades with onset of the most acute attack of symptoms in the morning hours. ${ }^{2}$ The collagen deposition, endothelial cell breakdown, and thickening of the trabecular meshwork causes extracellular matrix to project posteriorly as excrescences called guttae, one of the most recognizable characteristics of the disease. Symptomatic treatment options include hypertonic $\mathrm{NaCl}$ drops which draw water out of the edematous cornea. Definitive treatment requires corneal transplantation and it is estimated that FED patients account for one third of all corneal transplant patients.

Although the etiology of FED has not been conclusively determined, oxidative stress and apoptosis are most often cited as etiologic to the disease process. Borderie et $\mathrm{al}^{3}$ and Li et $\mathrm{a}^{4}$ reported apoptosis and apoptotic markers in corneal endothelium, stroma, and epithelium of FED patients. Since endothelial cells do not regenerate, long-term cell damage can lead to irreversible loss in the endothelium, ultimately causing the symptoms seen in FED. Wang et $\mathrm{al}^{5}$ demonstrated that increased exposure to oxidative stress may occur from a young age and that prolonged exposure combined with a decrease in antioxidants could lead to the endothelial cell damage seen in FED.

Glaucoma is a term used to describe a broad number of ocular disease processes. Most involve elevated intraocular pressure (IOP) and all can lead to optic nerve damage and progressive vision loss if not treated early. Glaucoma is primarily a clinical diagnosis based upon a number of criteria such as cup-to-disk ratio, visual field defects, and the general appearance of the optic nerve. The five major subtypes we address are ocular hypertension (OHT), glaucoma suspect (GS), primary open angle glaucoma (POAG), narrow angle glaucoma (NAG), and angle closure glaucoma (ACG). Treatment of glaucoma is centered on maintaining a lower IOP through topical medications or laser/surgical intervention.

The pathophysiology of glaucoma is composed of a wide range of genetic and environmental factors, but one commonly proposed mechanism between FED and glaucoma is oxidative stress. Agarwal et $\mathrm{ll}^{6}$ reported an increase in oxidative stress and apoptosis leading to retinal ganglion cell loss as one possible etiology of glaucoma, along with vascular insufficiency, cell excitotoxicity, and a change in the trabecular meshwork. This shared etiology between FED and glaucoma has led to the theory that there may be a common genetic predisposition to both diseases and that the prevalence of glaucoma may be higher in FED patients.

The purpose of this study was to use clinical data to determine if there is an association between FED and glaucoma patients by retrospective chart review.

\section{Methodology}

This was a retrospective cohort study with data collected from a private ophthalmology practice in Kansas City, MO, USA. Data was compiled into Excel from paper charts and electronic medical records. Patients were selected by searching for the International Classification of Diseases, Ninth Revision, Clinical Modification codes associated with FED and each of the subsets of glaucoma. FED patients were excluded from the data analysis if they had a history of Descemet's Stripping with Endothelial Keratoplasty (DSEK) or other corneal transplant procedures. A total of 257 FED patients were selected consecutively for the study. The FED patients were all Caucasian with ages that ranged from 42 to 96 years old with a mean age of 71 years old. Control patients were selected using a randomized list of patients seen in the clinic from January, 2011 to December, 2011. A total of 584 randomly selected controls with no history of endothelial dystrophy were included. Patients were excluded from the control group if they had any history of endothelial dystrophy, DSEK, or other corneal transplant procedure. Control patients were all Caucasian in order to be a more accurate comparison to the FED patients and the ages of the controls ranged from 42 to 95 years old with a mean of 68 years old. FED and control patients had similar mean IOP measurements of 16.3 and 16.2 mmHG respectively with a range of 8 to $38 \mathrm{mmHG}$. Sex distribution was also similar between the two groups with $40 \%$ males and $60 \%$ females in the control group compared to $34 \%$ males and $66 \%$ females in the FED patients. Exempt status of this study was approved through the Kansas City University of Medicine and Biosciences Institutional Review Board, Kansas City, Missouri, USA.

The patients were all examined by a board certified ophthalmologist trained in diagnosing and treating corneal diseases. Diagnosis of both FED and glaucoma/OHT was made by clinical diagnosis combining results of visual field testing with other clinical data. Other data parameters collected were age, sex, presence of diabetes, number of guttae, and IOP. Severity of FED was classified using number of guttae as category 1 , trace to $2+$ guttae; and category $2,3+$ to $4+$ guttae. 
Table I Prevalence of glaucoma combined and separated into GS/OHT and OAG in FED and control patients

\begin{tabular}{lllll}
\hline & No glaucoma & Glaucoma diagnosis & GS/OHT & OAG \\
\hline FED patients, $n(\%)$ & $160(62.3 \%)$ & $97(37.84 \%)$ & $56(21.86 \%)$ & $41(16.0 \%)$ \\
Controls, $n$ (\%) & $347(59.42 \%)$ & $237(40.68 \%)$ & $153(26.25 \%)$ & $84(14.5 \%)$ \\
\hline
\end{tabular}

Abbreviations: GS/OHT, glaucoma suspect/ocular hypertension; OAG, open angle glaucoma; FED, Fuchs' endothelial dystrophy.

\section{Statistical methods}

Based on the number of patients in the study, the statistical power this study provided was 0.893 for the result of presence or absence of glaucoma diagnosis and 0.983 for the result of the specific types of glaucoma. Models were generated in SPSS 9.0 (IBM Corporation, Armonk, NY, USA) using binomial and multinomial logistic regressions with generalized estimating equations (GEE). GEE was used because both eyes were used for most of the patients, requiring an adjustment for multiple data points from the same subject. Multiple binomial and multinomial regressions were done in a backward stepwise manner using linear and logistic regression respectively. Results included $P$-values and odds ratios (ORs) with confidence intervals (CIs) as presented in the Results section.

\section{Results}

There was no statistically significant difference in the overall prevalence of glaucoma in FED patients versus controls. The prevalence of glaucoma was $37.4 \%$ in FED patients compared with $40.8 \%$ in control patients. When the patients diagnosed with glaucoma were divided up into GS/ OHT patients and open angle glaucoma (OAG) patients, there was still no statistically significance difference in prevalence between either group in FED patients versus controls (Table 1). This prevalence of OAG is much higher than the measured population prevalence of OAG of $8.5 \%$ in Caucasian patients from the Salisbury eye study, ${ }^{7}$ but is not significantly different from the controls in our study.

There was no statistically significant difference in glaucoma prevalence in FED patients with either category 1 or
2 disease based on number of guttae ( $P=0.266$ and $P=0.086$, respectively). There was also no significant difference in glaucoma prevalence based on presence of diabetes or male sex ( $P=0.586$ and $P=0.066$, respectively). There was a statistically significant positive correlation in increasing glaucoma prevalence with increasing age and IOP $(P<0.001$ and $P<0.001$, respectively). This correlation remained significant in a multiple stepwise binomial regression with $P<0.001$ and $P<0.001$, respectively, for increasing age and IOP (Table 2).

There was a statistically significant difference in glaucoma prevalence when separated into GS/OHT and OAG in category 2 FED patients, but the correlation was negative meaning the prevalence of OAG was actually lower in patients with more advanced FED $(P=0.021)$. In category 1 FED patients, there was no significant difference in prevalence of the subtypes of glaucoma compared with controls $(P=0.092)$. There was also no significant difference in glaucoma prevalence based on presence of diabetes ( $P=0.745$ ), but there was a significant positive correlation between male sex and the presence of more severe subtypes of glaucoma ( $P=0.013)$. Similar to the model analyzing the prevalence of glaucoma as a whole, there was a statistically significant difference in glaucoma subtype prevalence with increasing age and IOP $(P<0.001$ and $P<0.025$, respectively) (Table 3).

Using a multiple multinomial logistic regression model, increasing age, increasing IOP, and male sex retained a significant positive correlation $(P=0.000,0.001$, and 0.000 , respectively) with OAG compared to less advanced GS/

Table 2 Binomial logistic regression using GEE with outcome of glaucoma diagnosis and null hypothesis as absence of glaucoma

\begin{tabular}{llllll}
\hline & Reference & Lower Cl & Upper Cl & B/OR & P-value \\
\hline Age & Scale & 0.008 & 0.025 & $0.017 / 1.017$ & $<0.001$ \\
Sex (male) & Sex (female) & -0.013 & 0.407 & $0.197 / 1.217$ & 0.066 \\
FED & Controls & -0.562 & 1.445 & $0.442 / 1.555$ & 0.389 \\
Diabetes & No diabetes & -0.321 & 0.182 & $-0.070 / 0.932$ & 0.586 \\
IOP & Scale & 0.020 & 0.075 & $0.047 / 1.048$ & 0.001 \\
Category I FED: trace to 2+ guttae & Controls & -1.597 & $0.44 I$ & $-0.578 / 0.561$ & 0.266 \\
Category 2 FED: 3+ to 4+ guttae & Controls & -2.007 & 0.134 & $-0.937 / 0.39$ & 0.086 \\
\hline
\end{tabular}

Abbreviations: GEE, generalized estimating equations; $\mathrm{Cl}$, confidence interval; $\mathrm{B} / \mathrm{OR}$, binomial divided by odds ratio; FED, Fuchs' endothelial dystrophy; IOP, intraocular pressure. 
Table 3 Multinomial logistic regression using GEE with outcome of GS/OHT and OAG versus no glaucoma

\begin{tabular}{llllll}
\hline & Reference & Lower Cl & Upper CI & B/OR & P-value \\
\hline Age & Scale & 0.014 & 0.031 & $0.022 / 1.022$ & $<0.001$ \\
Sex (male) & Sex (female) & 0.053 & 0.457 & $0.255 / 1.291$ & 0.013 \\
FED & Controls & -0.241 & 1.725 & $0.742 / 2.100$ & 0.139 \\
Diabetes & No diabetes & -0.285 & 0.204 & $-0.040 / 0.961$ & 0.745 \\
IOP & Scale & 0.004 & 0.057 & $0.030 / 1.030$ & 0.025 \\
Category I FED: trace to 2+ guttae & Controls & -1.858 & 0.140 & $-0.859 / 0.433$ & 0.092 \\
Category 2 FED: 3+ to 4+ guttae & Controls & -2.291 & -0.189 & $-1.24 / 0.289$ & 0.021 \\
\hline
\end{tabular}

Abbreviations: GEE, generalized estimating equations; GS/OHT, glaucoma suspect/ocular hypertension; OAG, open angle glaucoma; Cl, confidence interval; FED, Fuchs' endothelial dystrophy; IOP, intraocular pressure; B/OR, binomial divided by odds ratio.

OHT. There was no significant correlation either positive or negative between increasing severity of FED category with $\mathrm{OAG}$ as there was in the single multinomial regression.

\section{Conclusion}

The possibility of a connection between FED and glaucoma has been addressed in past literature with mixed results. This study complements previous studies which have been based on either a small number of study eyes or a glaucoma diagnosis by physician led questionnaire. Since the diagnosis of both glaucoma and FED was confirmed clinically, the results are more applicable to what ophthalmologists would see in the clinic on a daily basis. Our results showed no significant difference in prevalence of either glaucoma or its subtypes of GS/OHT or OAG in FED patients. We also found no significant positive correlation with glaucoma prevalence based on the severity of FED as was previously found by the Fuchs' Genetics Multi-Center Study Group. ${ }^{8}$

Previous studies demonstrate conflicting evidence on the association between FED and OAG. Buxton et $\mathrm{al}^{9}$ and Kolker and Hetherington ${ }^{10}$ reported a connection between corneal guttae of FED and POAG based on a lower facility of outflow and an elevated IOP/outflow facility ratio. Burns et $\mathrm{al}^{11}$ challenged these findings and in some cases ${ }^{12,13} \mathrm{dem}$ onstrated a negative relationship between corneal guttae from FED and elevated IOP. One proposed reason for the disputing evidence is that the increased corneal thickness in FED leads to a falsely low IOP. ${ }^{14}$ Recently Nagarsheth et $\mathrm{al}^{8}$ used a large study of Fuchs' genetics data and found a significant positive correlation between the severity of FED and an increased prevalence of OAG/OHT. Their study has been the largest to date analyzing the correlation between FED and OAG/OHT, but had several limitations as clearly stated by the authors including glaucoma being diagnosed by a physician led survey. ${ }^{8}$ Our study results conflict with the results of that study in regard to increased prevalence of glaucoma in more advanced FED patients based on corneal guttae and edema. This discrepancy in data may be caused by using a different population from a clinic setting versus a population recruited for genetic study. It also may be the result of the different method of diagnosing glaucoma. Our study used physician point-ofcare diagnosis based on clinical judgment compared with a physician led survey.

We had similar results to Nagarsheth et $\mathrm{al}^{8}$ in regard to age and IOP with a statistically significant increase in glaucoma prevalence with increases in either one. No other factors including sex, presence of diabetes, or FED diagnosis were statistically significant in relation to overall glaucoma prevalence in our study.

One unique aspect of our study was the ability to analyze different subtypes of glaucoma including GS/OHT and OAG. When these diagnoses were factored separately with OAG being considered the more severe condition, there was a significant positive correlation with age, IOP, and male sex. This result was maintained in multiple regression with all factors being considered together. There was still no significant connection with FED diagnosis or diabetes. There was a significant negative association between the presence of increasing glaucoma severity and increasing FED severity in single multinomial regression, but this result was not statistically significant on multiple multinomial regression. Thus our study showed no significant correlation between severity of FED and OAG as compared to GS/OHT, but this result may be caused by confounding factors and some of the limitations of our study.

The strengths of our study include a large sample size of 841 patients and direct clinical diagnosis of glaucoma by an ophthalmologist. One major limitation of our study is missing patient data limiting our ability to use several important variables, such as central corneal thickness due to not being available in many of the patients. Another important limita- 
tion is that our study population is from patients seen in clinic by a cornea-fellowship trained ophthalmologist and therefore the results might not be applicable to the general population. Also, many of the patients with more severe FED had already undergone corneal transplantation and therefore those eyes were excluded from the study leading to the possibility that our study underrepresented advanced cases of FED. This may explain the discrepancy between our results and Nagarsheth et al. ${ }^{8}$ The population of their study was heavily represented by patients with more advanced FED and therefore may have better addressed the effect of advanced FED on glaucoma prevalence.

Never the less, there are some common themes with the rest of the literature. It does not appear that there is a statistically significant association between FED and OAG prevalence, even when glaucoma patients are divided into GS/OHT and OAG patients. However there is still controversy regarding whether there is a connection between more advanced FED and OAG, and between FED and other forms of glaucoma such as narrow angle glaucoma. Prospective studies are needed to better elicit a connection between FED and glaucoma, and to determine if FED patients should undergo more regular glaucoma screening to improve early treatment of glaucoma and decrease the very preventable vision loss associated with glaucoma.

\section{Disclosure}

Dr Silverstein, Dr Wright, and Greg D Rice have no conflicts of interest in this work.

\section{References}

1. Pitts JF, Jay JL. The association of Fuchs's corneal endothelial dystrophy with axial hypermetropia, shallow anterior chamber, and angle closure glaucoma. Br J Ophthalmol. 1990;74(10):601-604.

2. Adamis AP, Filatov V, Tripathi BJ, Tripathi RC. Fuchs' endothelial dystrophy of the cornea. Surv Ophthalmol. 1993;38(2):149-167.

3. Borderie VM, Baudrimont M, Vallee A, Ereau TL, Gray F, et al. Corneal endothelial cell apoptosis in patients with Fuchs' dystrophy. Invest Ophthalmol Vis Sci. 2000;41(9):2501-2505.

4. Li QJ, Ashraf FM, Shen D, Green RW, Satrk WJ, et al. The role of apoptosis in the pathogenesis of Fuchs endothelial dystrophy of the cornea. Arch Ophthalmol. 2001;119(11):1597-1604.

5. Wang Z, Handa JT, Green WR, Stark WJ, Weinberg RS, et al. Advanced glycation end products and receptors in Fuchs' dystrophy corneas undergoing Decemet's stripping with endothelial keratoplasty. $O p h$ thalmology. 2007;114(8):1453-1460.

6. Agarwal R, Gupta SK, Agarwal P, Saxena R, Agrawal SS. Current concepts in the pathophysiology of glaucoma. Indian J Ophthalmol. 2009;57(4):257-266.

7. Friedman DS, Jampel HD, Muñoz B, West SK. The prevalence of open-angle glaucoma among blacks and whites 73 years and older: the Salisbury Eye Evaluation Glaucoma Study. Arch Ophthalmol. 2006; 124(11):1625-1630.

8. Nagarsheth M, Singh A, Schmotzer B, et al. Fuchs' Genetics MultiCenter Study Group. Relationship Between Fuchs Endothelial Corneal Dystrophy Severity and Glaucoma and/or Ocular Hypertension. Arch Ophthalmol. 2012;130(11):1384-1388.

9. Buxton JN, Preston RW, Riechers R, Guibault N. Tonography in cornea guttata. Arch Ophthalmol. 1967;77(5):602-603.

10. Kolker AE, Hetherington J. Becker-Shaffer's Diagnosis and Therapy of the Glaucomas. 4th ed. St. Louis: Mosby; 1976.

11. Burns RR, Bourne WM, Brubaker RF. Endothelial function in patients with cornea guttata. Invest Ophthalmol Vis Sci. 1981;20(1):77-85.

12. Krachmer JH, Purcell JJ, Young CW, Bucher KD. Corneal endothelial dystrophy: a study of 64 families. Arch Ophthalmol. 1978;96(11): 2036-2039.

13. Magovern M, Beauchamp GR, McTigue JW, Fine BS, Baumiller RC. Inheritance of Fuchs' combined dystrophy. Ophthalmology. 1979; 86(10):1897-1923.

14. del Buey MA, Cristobal JA, Ascaso FJ, et al. Biomechanical properties of the cornea in Fuchs' corneal dystrophy. Invest Ophthalmol Vis Sci. 2009;50:3199-3202.
Clinical Ophthalmology

\section{Publish your work in this journal}

Clinical Ophthalmology is an international, peer-reviewed journal covering all subspecialties within ophthalmology. Key topics include: Optometry; Visual science; Pharmacology and drug therapy in eye diseases; Basic Sciences; Primary and Secondary eye care; Patient Safety and Quality of Care Improvements. This journal is indexed on

Submit your manuscript here: http://www.dovepress.com/clinical-ophthalmology-journal

\section{Dovepress}

PubMed Central and CAS, and is the official journal of The Society of Clinical Ophthalmology (SCO). The manuscript management system is completely online and includes a very quick and fair peer-review system, which is all easy to use. Visit http://www.dovepress.com/ testimonials.php to read real quotes from published authors. 